\title{
Thrombolysis as an emergency treatment for a thrombosed prosthetic mitral valve diagnosed by transoesophageal echocardiography
}

\author{
Peter Currie, George R Sutherland, Ian R Starkey
}

\begin{abstract}
Intravenous streptokinase was used as an emergency treatment for acute thrombotic obstruction of a Björk-Shiley prosthesis in the mitral position. Transoesophageal echocardiography established the diagnosis and was used to monitor the clinical response to treatment. Because the patient was haemodynamically stable after thrombolysis and because transoesophageal echocardiography showed that the prosthesis was free of thrombus the mitral prosthesis was not replaced.
\end{abstract}

(Br Heart f 1993;70:198-200)

The incidence of acute prosthetic valve thrombosis in patients not taking anticoagulation may be as high as $8 \cdot 1 \%$ per patient year ${ }^{1}$ and the mortality associated with the conventional treatment for this condition-emergency reoperation-has been reported to be as high as $55 \% .^{2}$ Several reports have described the use of thrombolysis for the treatment of such patients ${ }^{3-5}$ but before thrombolysis is used it is important both to be able to make an accurate diagnosis and to monitor the clinical response because thrombosis may recur in a fifth of patients treated by thrombolysis. ${ }^{4}$ Doppler ultrasound can detect obstruction of prosthetic valves but transthoracic cross sectional echocardiography is poor at visualising mechanical prosthetic function and left atrial thrombus. Cineradiography can show reduced valve motion but does not visualise thrombus. A recent report described the potential use of transoesophageal echocardiography for the diagnosis and monitoring of such a case. ${ }^{6} \mathrm{We}$ describe a case of acute thrombotic obstruction of a Björk-Shiley mitral prosthesis in which the diagnosis was made and the response to thrombolytic treatment was monitored by transoesophageal echocardiography.

Department of Cardiology, Western General Hospital, Edinburgh P Currie G R Sutherland I R Starkey Correspondence to: Dr P Currie, Department of Cardiology, Western General Hospital, Crewe Road, Edinburgh, EH4 2XU. hemiparesis which fully resolved. Before admission she was taking daily doses of digoxin $(0.25 \mathrm{mg})$, frusemide $(160 \mathrm{mg})$, potassium chloride $(3.6 \mathrm{~g})$, and warfarin (variable dose).

On examination she was centrally cyanosed and tachypnoeic, with extensive pitting oedema of the lower limbs and considerable jugular venous engorgement. She was in atrial fibrillation (120 beats/min) with a supine blood pressure of $140 / 80 \mathrm{~mm} \mathrm{Hg}$. There were widespread coarse inspiratory crackles in the chest. Prosthetic valve clicks sounded normal with no murmurs. She was oliguric and had passed only $100 \mathrm{ml}$ of urine in 13 hours on the day of transfer. The chest radiograph showed pulmonary oedema and cardiomegaly.

Transthoracic cross sectional echocardiography showed a left ventricle of normal size with good systolic function; a markedly dilated right ventricle with prominent paradoxical septal motion but no clear abnormality of the mitral prosthesis. Doppler ultrasound showed an increased peak transmitral flow velocity $(3.0 \mathrm{~m} / \mathrm{s})$ with prolonged pressure half time indicating some obstruction of the prosthetic valve. There was severe tricuspid reflux, with an estimated pulmonary artery pressure of 70 $\mathrm{mm} \mathrm{Hg}$.

Transoesophageal echocardiography showed striking spontaneous echo contrast with virtually static flow in the left atrium with thrombus formation within the left atrial appendage and on the lateral left atrial wall (fig 1). The mitral prosthetic disc was immobile and was fixed by thrombus in the partly open position (fig 2). The findings were diagnostic of acute thrombotic obstruction of the mitral prosthesis. The diagnosis was supported by the finding that since operation anticoagulation had been suboptimal with a maximum prothrombin ratio of only 1.6 .

Within four and a half hours of admission the patient became moribund and was transferred to the intensive care unit for haemodynamic monitoring and inotropic support. The cardiac surgeons thought that she would not survive emergency surgery and she was therefore treated with a streptokinase infusion (500 000 IU over 30 minutes followed initially by $100000 \mathrm{IU} / \mathrm{h}$ ).

Six hours after the start of thrombolysis she remained oliguric and the dose of streptokinase was increased to $150000 \mathrm{IU} / \mathrm{h}$. Ten hours after the start of thrombolysis the urine output had increased and the peak transmitral Doppler velocity had fallen to below and three and a half weeks before admission her stenotic aortic valve was also replaced with a Björk-Shiley valve. Her postoperative course had been complicated by a mild left 


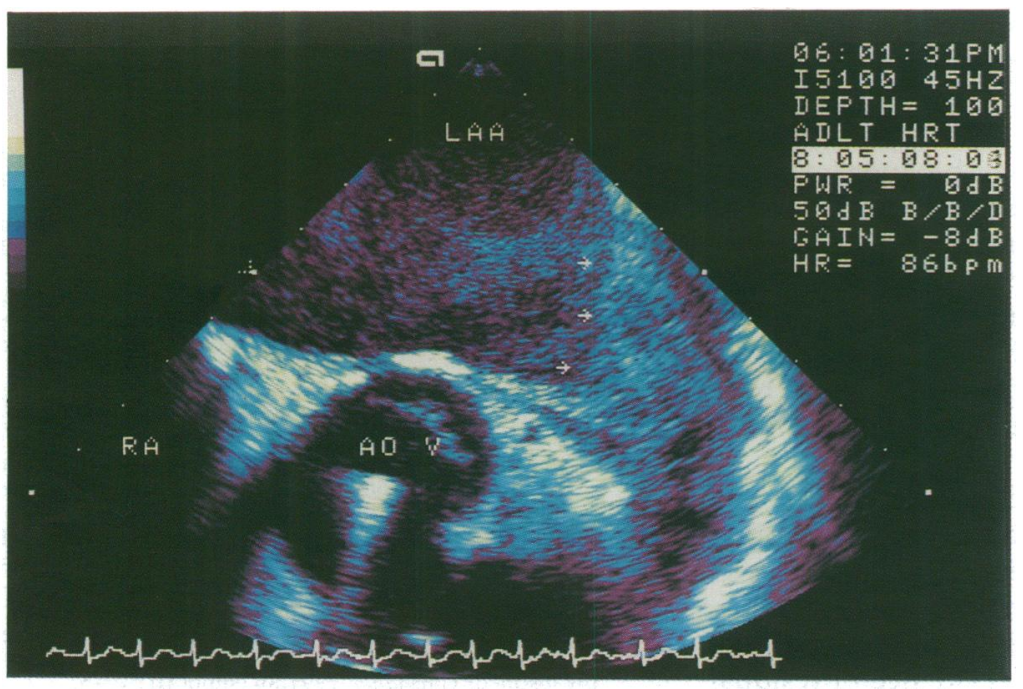

Figure 1 Transverse plane transoesophageal echocardiographic study of flow in the left atrium in a patient with an obstructed mitral valve prosthesis. There is spontaneous echo contrast in the left atrium indicating virtual stasis of blood. There is fresh thrombus (arrowed) in the left atrial appendage. AOV, Björk-Shiley aortic valve; $L A A$, left atrium and appendage; $R A$, right atrium.

$1.0 \mathrm{~m} / \mathrm{s}$. Transoesophageal echocardiography at 24 hours still showed reduced motion of the prosthetic disc but there was partial resolution of the left atrial thrombus and a reduction in spontaneous echo contrast in the left atrium.

The streptokinase infusion was continued for a total of three days without any haemorrhagic complications, embolic phenomena, or neurological sequelae. She was transferred back to the cardiothoracic surgical unit for a prosthetic mitral valve replacement but during the next three days she remained haemodynamically stable and transoesophageal echocardiography showed that the prosthesis

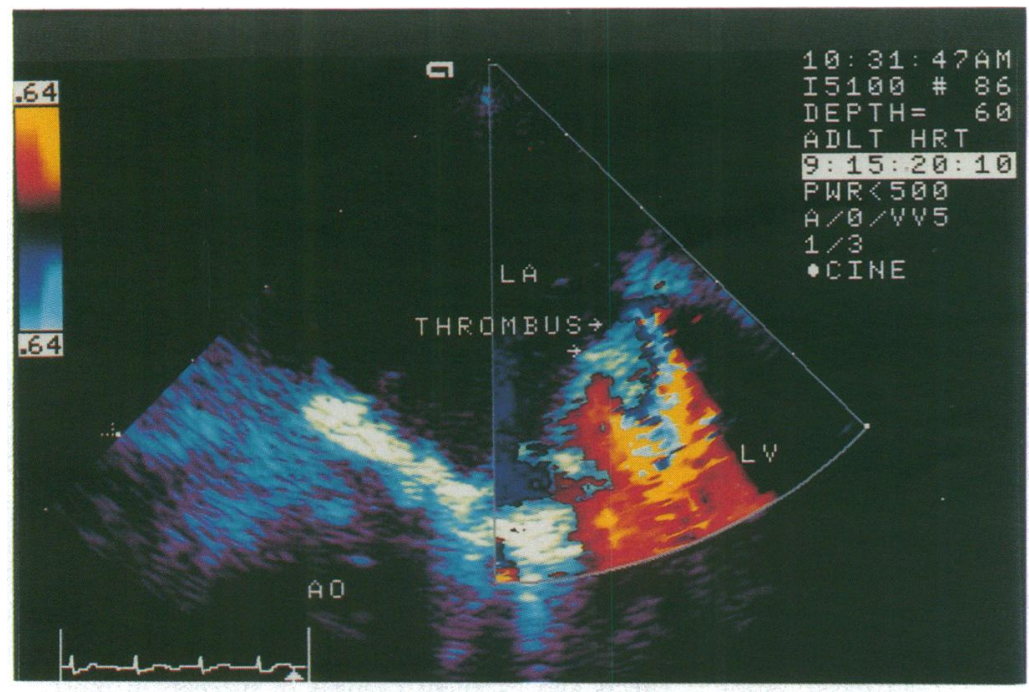

Figure 2 Transverse plane transoesophageal echocardiographic study in a patient with an obstructed Björk-Shiley mitral valve prosthesis. There is thrombus (arrowed) within the prosthesis which is obstructing blood flow through it. $A O$, aorta; $L A$, left atrium; $L V$, left ventricle. was free of thrombus: though a large thrombus was still present in the left atrial appendage. At this stage the cardiac surgeons considered that the risk of an early reoperation outweighed any possible benefit of surgery in a patient who was now haemodynamically stable. She was therefore maintained on warfarin (with a prothrombin time ratio in the range 2.5-3.5) for a further two weeks of observation at the Western General Hospital before being discharged home. Two days after discharge she was admitted to another hospital with a dense right hemiparesis and aphasia. A computed tomogram of the brain was not performed. She steadily deteriorated and died three weeks later.

\section{Discussion}

Mechanical prosthetic valves are liable to thrombosis and in this patient perioperative anticoagulation was inadequate-as it was in $60 \%$ of patients at the time of admission with thrombosis of a prosthetic heart valve. ${ }^{7}$ In our patient transoesophageal echocardiography was crucial in excluding mitral reflux, by demonstrating absence of prosthetic mitral valve motion and visualising the extensive thrombus in the left atrium and its appendage and on the mitral valve. Earlier precordial Doppler echocardiography had shown the abnormal transmitral flow velocity profile diagnostic of prosthetic obstruction.

Acute obstruction of the mitral prosthesis in our patient had several clinical features in common with other cases: inadequate anticoagulation, pulmonary oedema, oliguria, cyanosis, respiratory distress, and ineffectiveness of intravenous loop diuretics. ${ }^{46}$ The other striking feature in our patient was the rapid deterioration in her condition. Though prosthetic valve sounds were present the normal sounds of the coexisting Björk-Shiley aortic prosthesis probably masked the presumed absence of normal opening sounds of the mitral prosthesis.

Thrombolysis was life-saving in our patient and the risk of the alternative, emergency surgery, in this patient would have been very great. $^{2}$ The first signs of improvement appeared only after thrombolysis had been continued for over 10 hours; others have emphasised that prolonged therapy (that is, up to 72 hours) may increase the chance of success. ${ }^{34}$

More debatable is whether thrombolysis is a reasonable alternative to surgical intervention in less acutely ill patients. The mortality associated with acute reoperation for a second mitral prosthetic replacement was cited as $8 \%$ and $20 \%$ for patients in functional classes III and IV respectively ${ }^{2}$ while surgical thrombectomy alone carries much the same risk. ${ }^{7}$ Previous studies have shown that three quarters of patients gain at least a short-term improvement with thrombolysis ${ }^{4}$ while the risk of systemic embolisation during thrombolysis of left heart valve prostheses seems relatively small $(15 \%)$ and such emboli are rarely of clinical importance. ${ }^{4}$ However, 
others have reported recurrences of valve thrombosis in a fifth of patients initially successfully treated with thrombolysis and only about half of these recurrent thromboses can be successfully treated with further thrombolysis. ${ }^{4}$

In some patients it may be appropriate to use thrombolysis as a means of rendering a patient fit enough to undergo re-do surgery. In retrospect, this may have been a more appropriate management for our patient whose subsequent death, after initially successful thrombolytic treatment, may have been related to embolisation of persistent left atrial thrombus.

In critically ill patients who have had thrombosed prosthetic valves, we suggest that repeated transoesophageal echocardiography may be of great value in identifying patients with a low risk (no evidence of residual thrombus) or a high risk (evidence of residual thrombus, not necessarily directly related to the valve prosthesis, as in the reported case) of recurrent valve thrombosis. This will aid decisions about the need for further surgery.

1 Björk VO, Henze A. Ten years experience with the BjörkShiley tilting disc valve. F Thorac Cardiovasc Surg 1079; 78:331-42.

2 Husebye DG, Pluth JR, Peihler JM, et al. Reoperation on prosthetic heart valves: an analysis of risk factors in $\mathbf{5 5 2}$ patients. $\mathcal{F}$ Thorac Cardiovasc Surg 1983:4:543-52.

3 Ledain LD, Ohayon JP, Colle JP, Lorient-Roudaut FM, Besse PM. Acute thrombotic obstruction with disc valve prostheses: diagnostic considerations and fibrinolytic treatment. $\mathcal{F}$ Am Coll Cardiol 1986;7:743-51.

4 Kurzrock S, Singh AK, Most AS, Williams DO. Thrombolytic therapy for prosthetic cardiac valve thrombosis. F Am Coll Cardiol 1987;9:592-8.

5 Mehan VK, Dalvi BV, Kale PA. Streptokinase treatment of a thrombosed Björk-Shiley prosthesis in the aortic of a thrombosed Bjork-Shiley prosth

6 Young E, Shapiro SM, French WJ, Ginzton LE. Use of transoesophageal echocardiography during thrombolysis with tissue plasminogen activator of a thrombosed prosthetic mitral valve. $\mathcal{F}$ Am Soc Echocardiogr 1992;5:153-8.

7 Martinell J, Jimenez A, Rabago G, Artiz V, Fraile J, Farre J. Mechanical cardiac valve thrombosis. Is thrombectomy justified? Circulation 1991;84 (suppl III):70-5.

\section{ABSTRACTS IN CARDIOLOGY}

\section{Thrombolysis for throm- bosed prosthetic valves}

Thrombosis of a mechanical prosthesis is generally regarded as a surgical emergency because of the acute low output state which results. The case report from Currie et al (see above) shows that intravenous thrombolytic therapy can restore adequate function of the prosthesis.

Earlier this year Silber et al reported a prospective study from March 1978December 1991 in which they used thrombolysis as the initial treatment in 12 out of 17 patients with a thrombosed St Jude valve.
They used a combination of fluoroscopy and echocardiographic and Doppler studies to monitor the efficacy of the treatment. Two patients were treated with heparin, two with streptokinase, and the other eight with urokinase.

Surgery clearly still has a role: five patients in the series were managed with an operation rather than thrombolytic therapy.

Intriguingly, the case report from Currie et al raises the question whether elective surgery to remove persisting thrombus should be considered even when prosthetic function has been restored to normal by thrombolysis.

PETER MILLS

\section{The St Jude valve: thrombolysis as the first line of therapy for cardiac valve thrombosis}

Haim Silber, Steven S Khan, Fack M Matloff, Aurelio Chaux, Michele DeRobertis, Richard Gray

\section{Abstract}

Background-Thrombolytic therapy is a promising alternative to valve replacement in the management of prosthetic valve thrombosis. We sought to determine the short- and long-term results of treating thrombosed St Jude heart valves with thrombolytic therapy as the primary treatment modality.

Methods and results-Between March 1978 and December 1991, 988 patients underwent implantation of St Jude prosthetic valves at our medical centre, and all patients with thrombosed valves were identified prospectively. During this period, 17 patients (13 women; mean age $66.8 \pm$ 19.0 years) developed prosthetic valve thrombosis (11 aortic, six mitral). In six patients, Coumadin was stopped in preparation for elective surgery. The clinical presentation was congestive heart failure in 13, syncope and fatigue in two, and a cerebrovascular accident in one; one patient was asymptomatic. The average duration of symptoms was $11.7 \pm 12.0$ days (range $1-45$ days). Anticoagulation was subtherapeutic in all but one patient at the time of presentation. Cinefluoroscopy was the primary method used for diagnosis and was also used to follow the response to therapy. Twelve patients were treated medically (10 with thrombolytic therapy and two with heparin), three were treated surgically, and two were diagnosed at autopsy. Of the 12 medically treated patients, 10 had marked improvement in leaflet movement and symptoms within 12 hours. Thus, 10 of 12 patients $(83 \%)$ had a satisfactory response to medical therapy alone. No medically treated patient died or had a major complication resulting in permanent damage. However, four of the 12 medically treated patients had minor complications, including a transient episode of facial weakness in one patient, hematomas in two, and epistaxis in one. Late rethrombosis recurred in two patients in the medically treated group and was successfully retreated with thrombolytic therapy. At 3 months, all patients were alive and well.

Conclusions-Thrombolytic therapy can be used as the first line of therapy for thrombosed St Jude valves with a low risk of permanent side effects and excellent changes of success. In most cases, surgery can be reserved for patients who did not respond to thrombolytic therapy. (Circulation 1993;87:30-37.) 